\title{
ANÁLISIS Y EVALUACIÓN DE LOS NIVELES DE TOMA DE CONCIENCIA Y COMPRENSIÓN TÁCTICA EN FÚTBOL POR MEDIO DE UN TABLERO DE JUEGO
}

\section{ANALYSIS AND EVALUATION OF THE LEVELS OF AWARENESS \\ AND UNDERSTANDING OF FOOTBALL TACTICS BY A GAME BOARD}

\author{
Sánchez, R., Universidad Autónoma de Madrid • de la Vega-Marcos, Universidad Autónoma de Madrid \\ Ruiz-Barquín, R., Universidad Autónoma de Madrid • Del Valle, S., Universidad de Castilla la Mancha \\ Correspondencia: ricardo.delavega@uam.es \\ Recepción abril 2012 • Aceptación junio 2012
}

\section{RESUMEN}

El siguiente estudio aborda la relación existente entre el plano de acción y el plano de representación de los sujetos, a través del papel que proporcionan los procesos de comprensión y toma de conciencia que suceden en el interior del individuo cuando se enfrenta a situaciones de juego que demanda un deporte no lineal, con características sumamente abiertas, como es el fútbol. El objetivo que pretendemos es analizar y evaluar la toma de conciencia y comprensión táctica del jugador infantil de fútbol base, desde la perspectiva funcional-estructural. Para ello, se ha utilizado un juego de tablero simulador de fútbol, diseñado a partir de la reglamentación básica que rige este deporte. La muestra utilizada está compuesta por ocho sujetos varones de categoría infantil, divididos en dos niveles de pericia: alto y bajo. Por tanto, se trata de un análisis centrado en el jugador (Garganta, 2001). Los ítems utilizados para realizar este análisis de la comprensión táctica de los jugadores se pueden categorizar siguiendo los principios tácticos de juego en fútbol que detalla Claude Bayer (1987). A su vez, los contenidos tácticos que se detallan en los ítems, se clasifican en aspectos tácticos de tipo individual -ofensivo y defensivo-, grupal -ofensivo y defensivo- y colectivo -ofensivo y defensivo-, siguiendo las clasificaciones propuestas por Fradua (1997), Garganta y Pinto (1997), Sánchez y Fradua (2001).

Palabras clave: Educación Física, bilingüismo, inglés, educación primaria.

\section{ABSTRACT}

The following study addresses the relationship between the level of action and the level of representation of the subject, through the paper that provide the processes of understanding and awareness that occur within the individual when confronted with game situations sport demands a nonlinear, with features very open, like football. The objective we intend to analyze and evaluate the awareness and tactical understanding of children's football plaver base, from the structural-functional perspective. For this, we used a board game football simulator designed from the basic regulations governing the sport. The sample is composed of eight junior class male subjects, divided into two skill levels: high and low. Therefore, an analysis is centered on the player (throat, 2001). The items used for this analysis of tactical understanding of the players can be categorized according to the principles of tactical football game detailing Claude Bayer (1987). In turn, the contents listed in tactical items are classified in tactical aspects of individual type-offensive and defensive-, group-offensive and defensive-and collectiveoffensive and defensive-, following the classification proposed by Fradua (1997), Throat and Pinto (1997), Sanchez and Fradua (2001).

Key Words: Awareness, tactics, soccer, table game.

\section{INTRODUCCIÓN}

Se puede catalogar como interminable, la lista de autores que se han atrevido a escribir sobre el papel del juego a lo largo de estos últimos siglos, por lo que vemos necesario abordar tan sólo, aquellos planteamientos que se aproximen a las intenciones de la propuesta que presentamos.

Podemos comenzar manifestando que, hasta el siglo XX, el pensamiento que había dominado la opinión social y la educación, estaba marcado por la consideración del juego como una pérdida de tiempo, estimándose como una actividad realizada a partir del "gasto de energía sobrante", concepto que acuña Herbert Spencer (1855) a partir de su teoría de la energía sobrante. Gross (1889) a través de su teoría del preejercicio o autoeducación, basada en ideas darwinistas, se enfrentaba por primera vez a estos planteamientos poniendo en cuestión la inutilidad del juego, sin abandonar el concepto de energía sobrante. Gross se cuestionaba si un comportamiento tan ampliamente extendido en los animales más evolucionados, podría ser la base de la supervivencia. Por tanto, apostaba que las destrezas desarrolladas a partir del juego eran necesarias para la vida adulta, concibiendo el juego como un medio para desarrollar los instintos y la conducta inteligente. Mucho más interesante, si cabe, era su segunda idea, el origen de la capacidad de representación, que sería la referencia para la función simbólica. Gross tomaba al juego como una actividad con un objetivo capaz de diferenciarse de forma progresiva de las acciones que aparecen durante el juego, a partir del nivel de competencia que va adquiriendo el sujeto en su capacidad de representación. Estos estudios serían asumidos más tarde por Jean Piaget.

En oposición a la teoría de Gross, apareció la teoría de la dinámica infantil de Buytendijk $(1935,1948)$. Este autor cuestionaba la utilidad del juego en el desarrollo, afirmando que la maduración interna del individuo era la única responsable de este desarrollo. De este modo, según De la Vega, Del Valle, Maldonado y Moreno (2008) "la consideración de que el juego es el principal agente de la inmadurez infantil, constituye un precursor de la concepción de Bruner". No obstante, Buytendijk también apreciaba el juego, como un medio "fundamental a la hora de considerar la flexibilidad de las formas de conducta que permiten un mejor ajuste a la realidad". Sobre esta idea, Buytendijk realiza una aportación muy interesante sobre las características que debe reunir el juego. El autor relaciona el conocimiento y la novedad que debe contener el juego para el participante, es decir, afirma que el participante se muestra más interesado en aquellos objetos que le resultan en parte conocidos y que a su vez, abren nuevas posibilidades; y muestra rechazo hacia aquellos objetos que proponen situaciones absolutamente novedosas. Este planteamiento se acercaría a la concepción del juego que tiene Vigostky (1978) a través de su teoría de la Zona de Desarrollo Próximo. 
Por tanto, Gross y Buytendijk serían la base de las dos principales corrientes que estudian la psicología del juego. Como antes mencionamos, Piaget continuaría los estudios de Gross, mientras que Vigotsky haría lo propio con las investigaciones de Buytendijk.

La obra de Vigostky (1978), contiene una clara influencia de Marx, pues si para éste los instrumentos físicos nos permitían el control sobre el medio; para el primero, los instrumentos psicológicos permitirían modificar la mente. La gran diferencia entre Piaget y Vigotsky era la relación que establecen entre aprendizaje y desarrollo. Si para Piaget el desarrollo es la base de todo aprendizaje, para Vigotsky el desarrollo sigue al aprendizaje, es decir, sería el aprendizaje el que tirase del desarrollo y no a la inversa. De este modo, Vigotsky desarrolló su teoría llamada Zona de Desarrollo Próximo, en la que estableció la diferencia entre una zona de desarrollo actual, en la que el sujeto actúa autónomamente; y una zona de desarrollo potencial, en la cual, el sujeto puede adquirir su máximo nivel de competencia con la ayuda del adulto. $\mathrm{El}$ adulto puede utilizar el juego como medio de aprendizaje del niño o niña, pues según Vigotsky, el juego permite que el sujeto aprenda "a utilizar su imaginación (como proceso psicológico superior) que garantiza la realización de los deseos en un plano distinto al de la realidad”. Siguiendo a De la Vega, Del Valle, Maldonado y Moreno (2008) es necesario mencionar también a autores como Kurt Lewis (1935), Elkonin (1978), Linaza (1981), Maldonado (1981) y Ortega (1992), que reflexionan acerca de la utilización del juego en un marco real, respaldado por un marco irreal o imaginario; vaticinando la existencia de dos planos: el plano de acción y el plano de representación.

Por tanto, para Vigotsky los ejes centrales del juego son la situación imaginaria y las reglas, pues si el juego no se entiende sin situación imaginaria, tampoco puede entenderse sin reglas. Vigotsky analiza la necesidad de representar las distintas situaciones posibles, que se pueden generar para conseguir el objetivo, a partir de las reglas que el sujeto conoce. A medida que avanza esta capacidad de representación, la planificación de las acciones de juego, a través de la anticipación, toma un mayor sentido.

Por su parte, Piaget centra su investigación en la manera que el sujeto conoce la realidad, y afirma que la realidad no se presenta de forma uniforme para el sujeto, sino que está supeditada a su momento de desarrollo. Siguiendo a De la Vega, Del Valle, Maldonado, y Moreno (2008) podemos también mencionar, la gran importancia que otorga este autor a la coordinación de las acciones por parte del sujeto, como forma de aplicar sus operaciones mentales. A partir de estos hechos, Piaget muestra su interés por el juego, ya que reúne unas características muy interesantes para analizar la manera de entender que tiene el ser humano; debido a que, el sentido del juego "cambia para los niños de diferente edad. Así en los más pequeños el sentido está en las acciones y para los mayores el sentido está en las relaciones". (Garoz, 2002)

Por tanto, el marco de referencia para Piaget presenta un enfoque funcional (aprendizaje) y estructural (desarrollo), evidenciando una perspectiva funcional-estructural. Dicha perspectiva "postula la adquisición progresiva de niveles de conocimiento sobre las acciones. Se aboga por la importancia de la representación como elemento clave en la toma de conciencia", siendo lo más interesante de este planteamiento, "el poder conocer los diferentes niveles de toma de conciencia que tiene el sujeto con respecto a la acción para poder trabajar individualmente con él según sus propias necesidades. Por tanto, se parte de la función cognitiva para establecer el modelo de trabajo (entendido como estructura) para obtener mejoras cualitativas." (De la Vega, 2008:27).

Los enfoques presentados por Piaget y Vigotsky son relacionados por Bruner, mayor exponente de la psicología cognitiva, que también se plantea el juego como objeto de estudio. Su modelo teórico integra la acción, la emoción, el lenguaje y el pensamiento, considerando simultáneamente cada uno de estos elementos para interpretar la actividad lúdica. Cuando el sujeto consigue interpretar, en menor o mayor medida, esta relación de elementos, el juego se convierte en una actividad de exploración, que reduce la frustración ante el error, en contrastación con la situación real. La "consecuencia inmediata de esta disminución en las responsabilidades es la flexibilidad y riqueza combinatoria que se produce en la situación de juego, si en la actividad seria no caben las variaciones atrevidas ante la incertidumbre del resultado, en el juego esto, más que la excepción, constituye la norma”. Por tanto, el juego permite desarrollar la creatividad a través de la experimentación de acciones novedosas que no podrían ser realizadas en una situación real.

En la actualidad, el auge del juego tradicional de fútbolchapas como una práctica alternativa que cada vez cuenta con más adeptos, inspira una reflexión acerca de una posible adaptación del juego en búsqueda de unos objetivos que giren en torno a la comprensión de los fundamentos tácticos del fútbol real por parte de los participantes.

A partir de la utilización de las chapas como elemento motivante, este trabajo toma como referente principal una de las investigaciones que se presenta en De la Vega (2008), en la cual, se analiza la vinculación que presentan el plano de acción y el de la representación en el fútbol. Para hacerlo el autor diseña un juego de mesa denominado "Fútbol Inteligente".

Dicho juego, al igual que el juego que presentamos, se asienta sobre un modelo constructivista y desde una perspectiva funcional-estructural que postula la adquisición progresiva de niveles de toma de conciencia sobre las situaciones de juego. "El sujeto es considerado como un sujeto epistémico activo capaz de autorregular su representación y tener diferentes tipos de conocimiento: un conocimiento declarativo refiriéndose a los hechos; un conocimiento procedimental, relacionado con las reglas de acción; un conocimiento estratégico que combina las posibilidades de la acción siempre acorde con las reglas en una situación determinada; y un conocimiento metacognitivo que es la conciencia que tiene el sujeto respecto a los tres conocimientos anteriores" (De la Vega, 2008). De ahí la gran importancia, a la que hace mención Seirul-lo (1999) cuando nos transmite su línea general de entrenamiento para los deportes de equipo, en la cual, "interesa lo que sucede en el interior del deportista, después de que éste analice las condiciones que suceden a su alrededor, en el entorno, donde ha de realizar su actividad competitiva".

\section{MÉTODO}

\section{Participantes}

La muestra utilizada estuvo compuesta por ocho sujetos varones nacidos en el año 1997, por lo que en el momento de llevar a cabo la propuesta eran sujetos pertenecientes al primer año de la categoría infantil. No obstante, es necesario resaltar que los muestreos se realizaron en Junio, cuando la temporada llegaba a su fin. Podemos dividirlos a priori en dos niveles de pericia. Un grupo de alto nivel de pericia, compuesto por cuatro medio-centros del Rayo Vallecano S.A.D., equipo puntero del fútbol federado español; y un grupo de bajo nivel de pericia, compuesto por cuatro jugadores del C.F. Liceo Sport, club deportivo dedicado a la recreación y formación, cuyos sujetos formados en ligas municipales de fútbol sala y fútbol-7, no tuvieron todavía ningún contacto con el fútbol.

\section{Material}

Para la realización de este estudio contamos con varios instrumentos:

En primer lugar, el terreno de juego se representaba por medio de un tablero de coordenadas, por el que se desplazan las chapas numeradas, que representaban a los jugadores. A su vez, cada participante disponía de una pizarra de coordenadas y pivotes numerados, simulando el tablero de juego y las chapas, respectivamente. La estructura del tablero de juego, parte del planteamiento que ofrecen Garganta y Pinto (1997) y Jorge Castelo (1999; 2009), cuando dividen el terreno de juego en sectores (ofensivo, medio campo ofensivo, medio campo defensivo y defensivo) y carriles (izquierdo, central y derecho). Dicha relación nos va a permitir un acercamiento a la lógica interna del fútbol, a través de lo que llamaremos macrozonas de acción. A su vez, las macrozonas están formadas por un conjunto de zonas más 
pequeñas, que denominaremos "microzonas", que son identificadas por coordenadas, tal y como muestra el gráfico 1:

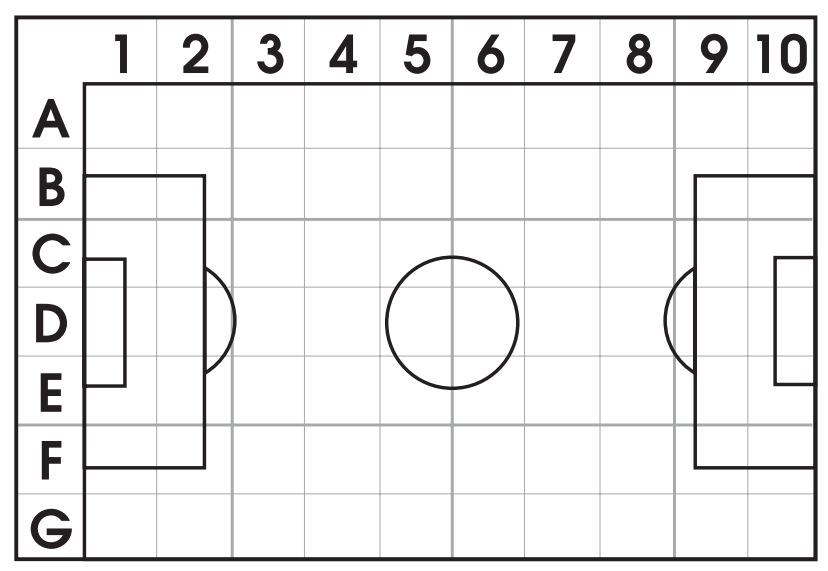

Gráfico 1: División del terreno de juego en zonas de actuación (macrozonas y microzonas)

Cada sujeto contaba, durante todo el juego (que tenía una duración de veinte turnos) con una pizarra en la que iba realizando sus movimientos y con la hoja de control de jugadores, donde se reflejaban los atributos y zonas de acción de los once jugadores, con el fin de facilitar el proceso a los participantes. Los turnos entre los dos participantes, se realizaban de una manera simultánea pues tal y como expone, De la Vega (2008): "el objetivo de realizar los movimientos de manera simultánea en vez de por turnos sucesivos, es el de simular la realidad del comportamiento táctico anticipatorio del fútbol, es decir, la posibilidad manifiesta de tener que anticipar cuáles son los movimientos ofensivos o defensivos que va a realizar el oponente para, de este modo, actuar en consecuencia con las “armas" de que disponen". Esto es algo que ya apuntaba, Josef Perner (1994) cuando habla de la capacidad de metarrepresentación refiriéndose a la capacidad que tiene el sujeto de representar las representaciones de las mentes de los demás. Por tanto, esta circunstancia resulta muy interesante en nuestra propuesta, pues como ya se ha mencionado, el participante debe anticipar sus movimientos a los movimientos que tiene previsto realizar su oponente.

Como es de suponer, las posiciones de los jugadores determinan sus zonas de actuación en el transcurso del partido. Tomando como referencia un estudio desarrollado por Castelo (1999:97), que analiza las distintas misiones tácticas de los jugadores en las diferentes zonas de actuación, de acuerdo al nivel de intervención de éstos sobre el balón; recogimos dichas zonas prioritarias de acción para cada posición, a partir de las macrozonas del tablero de juego, con el fin de perfilar la ficha de control de jugadores. También integramos en la ficha de control de jugadores, los atributos que caracterizarían a los distintos jugadores que componen el once de un equipo. De este modo, cada jugador según la posición tenía unas puntuaciones diferentes en los siguientes cinco atributos:

- Movimiento: Desplazamiento del jugador con o sin balón.

- Remate: Habilidad ofensiva del jugador para conseguir gol.

- Regate: Habilidad ofensiva del jugador en situaciones de oposición 1x1.

- Jugadas: Habilidad del jugador para pasar el balón.

- Defensa: Habilidad defensiva del jugador.

Para analizar las partidas en vivo, se utilizaba una hoja de observación en la que se recogían los movimientos que realizaban los participantes a lo largo del partido. A posteriori, se empleaba una hoja de postobservación para el análisis de la información recogida a través de las hojas de observación.

\section{Diseño}

Estamos ante un trabajo de investigación aplicada, cuyo diseño de investigación utilizado, se puede catalogar como "ex post facto prospectivo simple" (León y Montero, 2003), ya que comenzamos por seleccionar dos grupos de sujetos diferenciados por la variable independiente, para después determinar la posible influencia de ésta sobre las variables dependientes. En este caso, nuestra variable independiente (VI1) es el nivel de pericia que presentan los sujetos: alto o bajo. Las variables dependientes generales que recogen los resultados finales (puntuaciones de comprensión táctica) de cada sujeto analizado, son las siguientes:

- V.D.A: Puntuación obtenida en comprensión táctica individual ofensiva.

- V.D.B: Puntuación obtenida en comprensión táctica individual defensiva.

- V.D.C: Puntuación obtenida en comprensión táctica (colectiva) grupal ofensiva.

- V.D.D: Puntuación obtenida en comprensión táctica (colectiva) grupal defensiva.

- V.D.E: Puntuación obtenida en comprensión táctica colectiva ofensiva de equipo.

- V.D.F: Puntuación obtenida en comprensión táctica colectiva defensiva de equipo.

- V.D.G: Puntuación de comprensión táctica global

Estas variables generales actúan como variables categóricas, de las variables (ítems) encontradas en el instrumento de post-observación. Estas otras variables específicas quedan definidas y clasificadas de la forma siguiente:

Tabla 1: Relación de variables específicas y variables generales del estudio

\begin{tabular}{|c|l|}
\hline $\begin{array}{c}\text { Variables } \\
\text { generales }\end{array}$ & \\
\hline \multirow{4}{*}{ Variable DA } & Variable específicas \\
\cline { 2 - 3 } & Variable D4: Ítem 5: Usa la conducción con un jugador hábil \\
\cline { 2 - 3 } & Variable D5: Ítem 6: Busca profundidad en el pase. \\
\cline { 2 - 3 } & Variable D7: Ítem 8: Realiza un pase seguro. \\
\hline Variable DB & Variable D12: Ítem 13: Acosa/presiona al jugador con balón. \\
\hline \multirow{2}{*}{ Variable DC } & Variable D6: Ítem 7: E1 jugador que pasa, posteriormente se desmarca (pared) \\
\cline { 2 - 3 } & Variable D8: Ítem 9: Crea línea de pase con jugador que recibe el balón. \\
\hline
\end{tabular}




\begin{tabular}{|c|l|}
\hline \multirow{2}{*}{ Variable DD } & Variable D10: Ítem 11: Corta las posibles líneas de pase. \\
\cline { 2 - 2 } & $\begin{array}{l}\text { Variable D11: Ítem 12: Realiza coberturas sobre jugadores que pueden ser } \\
\text { sobrepasados. }\end{array}$ \\
\cline { 2 - 2 } & Variable D13: Ítem 14: Fuerza situaciones 1x2 en posible microzona objetivo. \\
\hline \multirow{2}{*}{ Variable DE } & Variable D1: Ítem 2: Número de jugadores que son desplazados \\
\cline { 2 - 2 } & Variable D2: Ítem 3: Distribución de los jugadores por zonas de acción \\
\hline Variable DF & $\begin{array}{l}\text { Variable D9: Ítem 10: Sitúa todos los jugadores por detrás (o en línea) de balón, cuando } \\
\text { el balón está en campo contrario. }\end{array}$ \\
\hline Variable DG & Promedio de las variables generales DA, DB, DC, DD, DE, DF, DG \\
\hline
\end{tabular}

\section{Procedimiento}

En primer lugar, se realizaba una breve explicación a los participantes sobre los beneficios que aporta el juego de tablero para analizar y evaluar la comprensión táctica. A continuación se utilizaba el video explicativo para que los sujetos conocieran el reglamento de juego, y a la vez entendieran cómo se jugaba. Dicho reglamento se expone de forma extensa y clara en Sánchez, R. (2010). Las dudas que iban surgiendo a través de la visualización del video, iban siendo resueltas por el experimentador.

Antes de comenzar el partido, se realizaban varias simulaciones de prueba, a partir de situaciones de juego tres contra tres. Cuando el observador, apreciaba que los sujetos tenían claras las nociones del juego, se procedía a disputar el partido. Como ya se ha explicado al describir el material empleado, mientras los sujetos toman parte del proceso de juego, el observador se dedica a recopilar los movimientos de juego que los sujetos realizan tanto en ataque, como en defensa. Los datos que contiene la hoja de observación son analizados a través de la hoja de post-observación obteniendo la información que buscamos para ser evaluada de acuerdo a los ítems que presenta la hoja (variables dependientes específicas).

Para el análisis de datos, se utilizaron las siguientes pruebas:

- Análisis de fiabilidad de las observaciones:

- Índice de acuerdo inter-jueces Kappa para variables dicotómicas o cualitativas.

- Acuerdo inter-jueces mediante el coeficiente de correlación de Pearson para variables cuantitativas.

- Análisis descriptivos de los resultados:

- Aplicación de las medidas de tendencia central (media) y dispersión (desviación típica) para las variables específicas cuantitativas.

- Realización de los análisis de frecuencias y porcentajes para las variables específicas cualitativas.

- Promedios para la obtención de las variables generales.

- Análisis de diferencias de medias para dos muestras independientes:

- Pruebas de chi-cuadrado para variables dicotómicas o cualitativas.

- Estadístico U de Mann-Whitney para variables cuantitativas.

\section{RESULTADOS}

Con el fin de comprobar la validez de la herramienta, optamos por realizar un proceso de fiabilidad, a través del criterio inter-jueces. Para ello, recurrimos a cuatro observadores externos, dos analizaron un partido de nivel alto de pericia, y los otros dos un partido de nivel bajo de pericia. Ambos partidos fueron determinados por azar. Para realizar dicho análisis, utilizaron la hoja de post-observación, con el fin de establecer la fiabilidad de sus variables. Para la contrastación de los resultados, se utilizó la medida de acuerdo kappa para determinar el índice de acuerdo de las variables que recogen datos cualitativos (frecuencia y porcentaje), obteniéndose un promedio global de 0,879 . Hay que tener en cuenta que, cuando nos enfrentamos a variables constantes, tuvimos que utilizar el índice de acuerdo entre jueces descrito en León y Montero (2003:96), ya que la medida de acuerdo kappa no permite trabajar con este tipo de datos. Por otro lado, para determinar la fiabilidad de las variables de tipo cuantitativo, se realizó la correlación de Pearson (medias y desviaciones típicas), con un resultado de 0,929 . Ambos índices, aseguran una fiabilidad muy alta de la herramienta.

Resumen de las puntuaciones obtenidas en las variables específicas del estudio

Las pruebas de contraste chi-cuadrado para variables cualitativas y U de Mann Whitney para variables cuantitativas, desvelaron que existen diferencias significativas en cinco (V.D.1., V.D.2, V.D.4, V.D.7, V.D.9) de las trece variables específicas estudiadas. (V.D.A)

Puntuación obtenida en comprensión táctica individual ofensiva

El valor promedio de las cuatro variables específicas (V.D.3, V.D.4, V.D.5, V.D.7) que conforman la puntuación de comprensión táctica individual ofensiva se sitúa en $67,7 \%$ para toda la muestra. Las diferencias entre los valores alcanzados por el grupo de alta pericia $(82,0 \%)$ y los valores del grupo de baja pericia $(55,4 \%)$ son muy significativas. (V.D.B)

Puntuación obtenida en comprensión táctica individual defensiva

En este caso, la variable general V.D.B. sólo cuenta con una sola variable específica (V.D.12), por lo que los valores obtenidos en dicha variable específica, pasan a extrapolarse para la variable general.

Los resultados obtenidos reflejan, unas puntuaciones bajas $(24,3 \%)$ de comprensión táctica asociada a aspectos tácticos individuales defensivos para toda la muestra, no apreciándose diferencias significativas entre los grupos de pericia.

Puntuación obtenida en comprensión táctica grupal ofensiva (V.D.C)

Los valores obtenidos a partir del promedio de las variables específicas V.D.6 y V.D.8, para la variable que refleja la puntuación en táctica grupal ofensiva (V.D.C.), nos muestran que el grupo de sujetos de baja pericia obtiene mejores puntuaciones $(70,9 \%)$ que el grupo de sujetos de alta pericia $(60,6 \%)$.

Puntuación obtenida en comprensión táctica grupal defensiva (V.D.D)

La siguiente variable se obtiene a partir de las variables específicas V.D.10, V.D.11 y V.D.12. Como se puede apreciar, las puntuaciones obtenidas para esta variable asociada a la táctica grupal defensiva (V.D.D.) son muy parecidas entre los dos diferentes grupos de análisis, obteniendo el grupo de alta pericia un 28,9\% y el grupo de baja pericia un $23,1 \%$.

Puntuación obtenida en comprensión táctica colectiva ofensiva de equipo (V.D.E)

Para poder obtener la puntuación de este ítem debemos transformar los valores que contienen dos variables de tipo cuantitativo (V.D.1 y V.D.2) en datos cualitativos. De este modo, la V.D.1 mide el número de movimientos que realiza cada sujeto por turno, aportándonos una media general de todos los turnos. Como el número de movimientos es sobre los diez jugadores de campo, podemos transformar la media en porcentaje. Es decir, si un sujeto mueve a 6 jugadores de sus 10 disponibles como media, moverá un $60 \%$. Con la V.D.2 vamos a hacer algo parecido. Tomando 
como referencia la media de jugadores posicionados en zonas de acción verde, que representaban las zonas de acción de equilibrio del equipo, sabremos el porcentaje de jugadores que hay en zonas verdes respecto al total de jugadores.

Se puede observar en los datos obtenidos que el grupo de sujetos de alta pericia alcanza un $67,7 \%$ de puntuación, mientras que los de baja pericia obtienen un 56,9\%.

Puntuación obtenida en comprensión táctica colectiva defensiva de equipo (V.D.F)

Cómo ya ocurriera, en la variable general V.D.B, la variable V.D.F sólo cuenta con una sola variable específica (V.D.9), por lo que los valores obtenidos en la variable general serán los mismos que en la variable específica.

Los resultados nos muestran diferencias muy significativas entre el grupo de alta pericia y el de baja pericia, alcanzando los primero una puntuación del $73,9 \%$, y situándose los segundos en el 21,9\%.

Puntuación de comprensión táctica global (V.D.G.)

La puntuación de comprensión táctica global, se calcula a partir del promedio de las puntuaciones del resto de variables dependientes generales. De este modo, se puede apreciar que el grupo de sujetos de alta pericia obtiene una puntuación global de $55,9 \%$, mientras que los sujetos de baja pericia consiguen una puntuación global de $42,1 \%$. Esto significa que existen diferencias significativas entre ambos grupos estudiados.

Resumen de puntuaciones

A continuación, se ofrece un resumen de las puntuaciones obtenidas en todas las variables generales (Gráfico 1 y tabla 2).

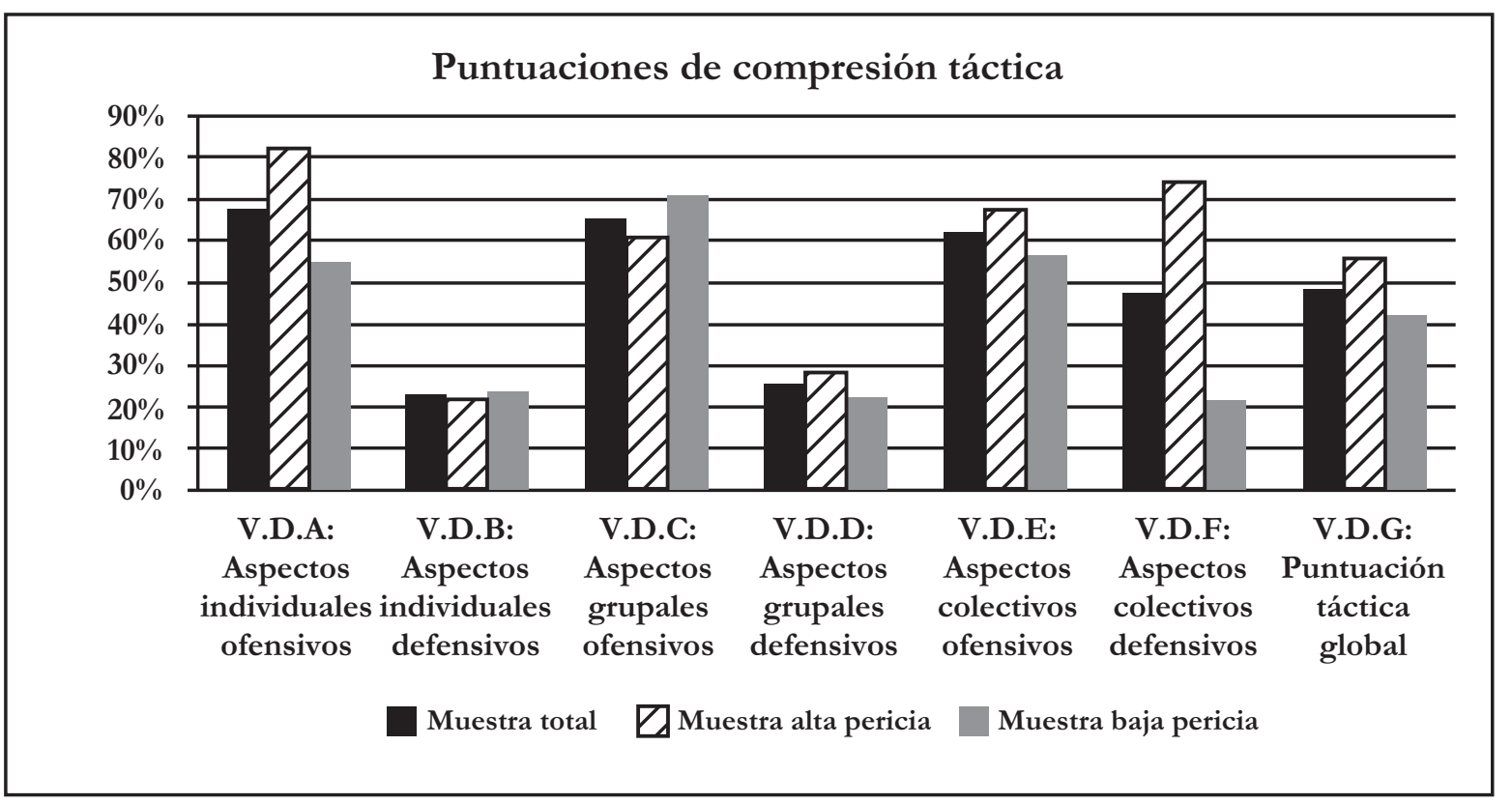

Gráfico 1. Niveles de comprensión táctica en las variables generales de estudio

Tabla 2.- Resumen de datos

\begin{tabular}{|l|c|c|c|}
\hline \multicolumn{1}{|c|}{ Variables dependientes generales } & Muestra total & $\begin{array}{c}\text { Muestra alta } \\
\text { pericia }\end{array}$ & $\begin{array}{c}\text { Muestra baja } \\
\text { pericia }\end{array}$ \\
\hline V.D.A: Aspectos individuales ofensivos & $67,7 \%$ & $82,0 \%$ & $55,4 \%$ \\
\hline V.D.B: Aspectos individuales defensivos & $23,4 \%$ & $22,2 \%$ & $24,3 \%$ \\
\hline V.D.C: Aspectos grupales ofensivos & $65,7 \%$ & $60,6 \%$ & $70,9 \%$ \\
\hline V.D.D: Aspectos grupales defensivos & $25,5 \%$ & $28,9 \%$ & $23,1 \%$ \\
\hline V.D.E: Aspectos colectivos ofensivos & $62,3 \%$ & $67,7 \%$ & $56,9 \%$ \\
\hline V.D.F: Aspectos colectivos defensivos & $47,8 \%$ & $73,9 \%$ & $21,9 \%$ \\
\hline V.D.G: Puntuación táctica global & $48,7 \%$ & $55,9 \%$ & $42,1 \%$ \\
\hline
\end{tabular}




\section{DISCUSIÓN}

El análisis de los resultados nos permitió conocer el nivel de comprensión táctica y toma de conciencia que tienen los sujetos analizados de categoría infantil, en función de su nivel de pericia. A partir de esta circunstancia, pudimos concretar más específicamente que, los sujetos de alta pericia presentan mejores resultados que los sujetos de baja pericia, en aspectos asociados a táctica individual y táctica colectiva de equipo. Sin embargo, no obtuvimos diferencias significativas entre ambos grupos, para las puntuaciones asociadas a táctica grupal, lo que vislumbra las debilidades que presentan los jugadores infantiles de fútbol analizados en estos aspectos tácticos, y conduce a replantearnos una vez más, la utilización de modalidades reducidas del juego del fútbol en categorías inferiores, tales como el fútbol 3, el fútbol-5, el fútbol-7 o el fútbol-9, compartiendo la idea de muchos otros autores, entre los que podemos destacar a Wein (1995), Lapresa y cols. (1999, 2001, 2006, 2008a, 2008b), Martínez y Solla (2009).

También pudimos comprobar en los sujetos analizados que, las puntuaciones alcanzadas en aspectos ofensivos son más elevadas que las puntuaciones que presentan en aspectos defensivos. Esto ocurre tanto en aspectos individuales, como en aspectos grupales y colectivos. Esta información no nos sorprende, ya que sabemos que, por norma general, se trabajan mucho más los aspectos de ataque, que los aspectos de defensa, en los entrenamientos ligados a las primeras etapas de formación; y hay que apuntar en este sentido, que la comprensión de los aspectos tácticos defensivos suele resultar más compleja que la comprensión de los aspectos tácticos ofensivos.

Parece necesario, especificar de alguna u otra forma las limitaciones, que desde nuestro punto de vista, presenta este estudio. En primer lugar, no tenemos en cuenta el nivel de inteligencia del sujeto. Este hecho puede sesgar ciertos datos debido a que la toma de conciencia está estrechamente ligada a la inteligencia del sujeto. Sería interesante en futuras investigaciones, la utilización de la prueba de matrices progresivas de Raven, con el fin de conseguir una muestra más homogénea, en cuanto a inteligencia se refiere. En segundo lugar, pensamos que a pesar de conseguir unas puntuaciones muy elevadas en el proceso de frabilidad inter-jueces, la hoja de post-observación resulta muy compleja y extensa, por lo que sería muy interesante poder crear un programa informático que realizará la labor de análisis, a partir de la introducción de los datos recogidos en la hoja de observación.

Por otra parte, como se ha podido apreciar a lo largo del proyecto, podemos concretar que el beneficio principal del proyecto, es verificar que existe la posibilidad de analizar y evaluar la comprensión táctica del sujeto a través del tablero de juego, como ya apuntaba De la Vega (2008).

\section{REFERENCIAS BIBLIOGRÁFICAS}

Bayer, C. (1987). La enseñanza de los juegos deportivos colectivos. Barcelona: Hispano Europea.

Buytendijk, F. J. J. (1935). El juego y su significado. Madrid: Revista de Occidente.

Buytendijk, F. (1948). Psicología del juego. Madrid: Gredos. Casteló, J. (1999). Fútbol: Estructura y dinámica del juego. Barcelona: INDE.

Casteló, J. (2009). Tratado general de fútbol. Guía práctica de ejercicios de entrenamiento. Barcelona: Editorial Paidotribo.

De la Vega, R. (2008). Pensamiento y acción en el deporte perspectiva funcional-estructural. Sevilla: Wanceulen.

De la Vega, R., Del Valle, S., Maldonado, A., \& Moreno, A. (2008). Una nueva herramienta para la comprensión táctica del fútbol. Revista Internacional de Medicina y Ciencias de la Actividad Física y el Deporte vol. 8 (30) pp. 130-145. En http:/ / cdeporte.rediris.es/revista/revista30/arttactica $72 . h t m$

Fradua, L. (1997). La visión de juego en el futbolista. Barcelona: Paidotribo.

Garoz, I. (2002). Juegos y deportes. Un estudio del Palin
Mapuche y el hockey hierba. Tesis Doctoral sin publicar. Madrid. UAM.

Garganta, J. (2001). Análisis del juego en el fútbol. Training Fútbol, 64, 14-27.

Garganta, J., \& Pinto, J. (1997). La enseñanza del fútbol. En A. Graca, y J. Oliveira, (Ed.) La enseñanza de los juegos deportivos (pp. 97-138). Barcelona: Paidotribo.

Groos, K. (1889). The play of animáis. New Cork: Appleton. Lapresa, D., Arana, J., \& Ponce de León, A. (1999). Orientaciones educativas para el desarrollo del deporte escolar. Logroño: Universidad de La Rioja y Federación Riojana de Fútbol.

Lapresa, D., Arana, J., \& Navajas, R. (2001). La alternativa del fútbol 9 en el primer año de infantiles: una propuesta ante el fútbol 11. Revista el Entrenador Español. 88: 34-41.

Lapresa, D., Arana, J., \& Garzón, B. (2006). El fútbol 9 como alternativa al fútbol 11, a partir del estudio de la utilización del espacio de juego. Apunts: Educación física y deportes. 85, 34-44.

Lapresa, D., Amatría, M., Egüén, R., \& Garzón, B. (2008). Análisis descriptivo y secuencial de la fase ofensiva del fútbol 5 en la categoría prebenjamin. Revista de Ciencias de la Actividad Física y del Deporte. Universidad Católica de San Antonio. 8: 107-116.

Lapresa, D., Arana, J., Garzón, B., Egüén, R., \& Amatria, M. (2008). Enseñando a jugar el fútbol. Hacia una iniciación coherente. Universidad de la Rioja y Federación Riojana de Fútbol. Logroño.

León, O., \& Montero, I. (2003). Métodos de investigación en psicología y educación. Madrid. Mc Graw Hill.

Martínez, M., \& Solla, J.J. (2009). Los modelos de competición en el fútbol en categorías de formación: fútbol 11, fútbol 7 y fútbol 5. En 1. Casáis, E., Domínguez, C. Lago, \& cols.. Fútbol base: El entrenamiento en categorías de formación. Volumen I. Pontevedra. MCSports.

Perner, J. (1994). Comprender la mente representacional. Barcelona: Paidos.

Sánchez, D.L., \& Fradua, L. (2001). La planificación en el fútbol base: modelo aplicativo de planificación integral. Training fútbol, 63, 26-41.

Sánchez, R. (2010). Fútbol chapas revolutions: Un juego de tablero como alternativa para trabajar la comprensión táctica en fútbol. Trabajo fin de Máster sin publicar. UAM.

Seirul·lo, F. (1999) Criterios modernos de entrenamiento del fútbol. Training Futbol, 45, 8-17

Spencer, H. (1855). The Principles of Psychology. Londres : Longman's.

Vigotsky, L. S. (1978). El desarrollo de los procesos psicológicos superiores. Barcelona: Grijalbo.

Wein, H. (1995). Fútbol a la medida del niño. Madrid: Real Federación Española de Fútbol.
REVISTA EUROAMERICANA DE CIENCIAS DEL DEPORTE

ISSN 2254-4070 / Vol. 1, nº1 / Murcia Julio 2012 / Págs. 33 - 38
Análisis y evaluación de los niveles de toma de conciencia y comprensión táctica en fútbol por medio de un tablero de juego. SportTK, 1[1], 33 - 38 